\title{
Influence Research of Wind Power Generation on Power System
}

\author{
Qiguo YAO ${ }^{a,{ }^{*}}$, Yuliang LIU ${ }^{\mathrm{b}}$
}

School of Naval Architecture \& Mechanical-electrical Engineering, Zhejiang Ocean University,
Zhoushan, Zhejiang, 316022, China

ayaoqiguo@163.com, blyl_zjou@126.com, ”corresponding author

Keywords: Wind Power Generation; Power System; Influence; Wind Farms

\begin{abstract}
Wind power generation is always weather dependent and has the trend of being integrated to power systems as the form of large-scale wind farms, which influences on power systems. Since the power network was not designed specifically to accommodate this type of generation, there are inevitably some points at which modifications must be executed if existing standards of electricity supply are to be maintained. This paper discusses in general terms the problems which are encountered by the developers of wind power generation projects and by utility grids when dealing with projects to integrate wind farms to power systems. The influence includes active and reactive power flow, voltage, system stability, power quality, short-circuit capacity, system reserve, frequency and protection due to the characteristics of high-capacity, dynamic and stochastic performance of wind power generation. Corresponding countermeasures to handle these issues are recommended in order to accommodate wind power generation in power systems.
\end{abstract}

\section{Introduction}

In renewable energy, wind energy has a strong competitiveness. Wind power increasingly mature in technology and commercial application, it is one of the most large-scale soon prospect of development and utilization among renewable resources. In economic aspect, wind power costs is lower, while the conventional energy generation costs further increase due to the requirements of environmental protection, and along with the technology improvement, the cost of wind power will have a further reduction.

Large-scale wind power must achieve parallel operation. The analysis of the wind farm connected to the power system is indispensable content to design and operation of a wind farm, it is also one of the three major issues of wind power technology. Although the European and American have some practical experience and technical regulations for construction and operation of wind power, but as a result, the actual situation of China's power structure is different from them, these practical experience and technical regulations are not entirely suitable for the situation of our country. This article mainly introduced the influence of wind power integration to power system.

\section{Characteristics of Wind Power Generation}

From the point of view of wind energy, the most striking characteristic of the wind resource is its variability. The stochastic variation of wind farms outputs root mainly in fluctuation of the wind speeds and directions. The wind is highly variable, both geographically and temporally. Furthermore this variability persists over a very wide range of scales, both in space and time [1].

From a power system perspective, the turbulent peak may affect the power quality of wind power generation. The influence of turbulences on power quality depends very much on the turbine technology applied. Variable-speed wind turbines, for instance, may absorb short-term power variations by the immediate storage of energy in the rotating masses of wind turbine drive trains. That means that the power output is smoother than strongly grid-coupled turbines, fixed-speed wind turbines. Diurnal and synoptic peaks, however, may affect the long-term balancing of power system, in which wind speed forecasts plays a significant role.

Another important issue is the long-term variations of the wind resources. The wind speed up to the height of the hub should be known to calculate the wind farm output. A number of 
measurements of wind speeds show that wind speeds are mostly mild in a year, their probabilities between 0 and $25 \mathrm{~m} / \mathrm{s}$ are considerable; most of the average annual wind speeds subject to the Weibull distribution [2], as in formula (1).

$$
f(v)=\frac{k}{c}\left(\frac{v}{c}\right)^{k-1} \cdot \exp \left(-\left(\frac{v}{c}\right)^{k}\right)
$$

where: $v$ is average wind speed; $k$ is shape parameter; $c$ is scale parameter.

The relationship between the wind turbine output $P_{w}$ and the wind speed up to the height of the hub $v$ can be expressed approximately as the curve of wind turbines' outputs vs. wind speed or a subsection function, as in formula (2).

$P_{w}= \begin{cases}0 & \left(v \leq v_{C I} \quad \text { or } \quad v \geq v_{C O}\right) \\ \frac{P_{R}}{v_{R}^{3}-v_{C I}^{3}} v^{3}-\frac{v_{C I}^{3}}{v_{R}^{3}-v_{C I}^{3}} P_{R} & \left(v_{C I} \leq v \leq v_{R}\right) \\ P_{R} & \left(v \geq v_{R}\right)\end{cases}$

where: $P_{w}$ is rated output of the wind turbine; $v$ is wind speed up to the height of the hub; $v_{C I}$ is cut-in wind speed; $v_{C O}$ is cut-out wind speed; $v_{R}$ is rated wind speed.

\section{Influence of Wind Power Generation on Power System}

High penetration of wind power in the power systems faces fundamental technical limits with regard to the integration of large-scale wind farms to the grid. The influence of wind power generation on power systems includes active and reactive power flow, voltage, system stability, power quality, short-circuit capacity, system reserve and infrastructure due to the characteristics of high-capacity, dynamic and stochastic performance of wind power generation. Technically, it influences the gird in the following ways and has to be studied in detail:

Active and Reactive Power Flow. The calculation of power flow including wind farms is often applied for evaluating the influence of wind power generation on power systems. The key point is how to deal correctly with the wind turbines and the node type of the point of common coupling (PCC).

Wind power is a kind of intermittent and stochastic power source, which will complicate the power flow. Because many wind farms are built far away from load centers in order to capture more wind energy, there is always some obstacle of transmitting wind power. Some transmission or distribution lines and other electrical equipments may be over-loaded when the additional wind power generation is introduced. So it should be ensured that the interconnecting transmission or distribution lines will not be over-loaded. Both active and reactive power requirements should be investigated. Reactive power should be generated not only at PCC, but also throughout the network, and should be compensated locally [3].

The methods utilized for analysis of conventional generators are certain and ignore the uncertainty of wind speed and load forecasts. Therefore, the probabilistic method is more suitable for wind power generation. This model is based on the wind speed distribution, such as formula (1). The constraints are described by probabilistic forms and the expected values of parameters, such as voltages and powers can be computed.

Voltage Regulation. Once a wind farm has identified its site, the point at which connection to the grid must be identified.

Small wind farm can connect at lower voltage, thereby saving on switchgear, cable and transformer costs. If the size of the proposed development is too large to be connected at the local distribution voltage, access to the transmission network at a higher voltage is required [4].

After failures, if the transient instability does not occur in power systems, some wind turbines shut down due to their low voltage protections. Then outputs of wind farms decrease, which means that the power system lose reactive loads. Therefore the voltage levels climb up, even beyond the upper limits of wind farms buses. 
Capacitors are the common reactive power compensation methods. When voltage levels drop down, the amount of compensation decreases much. However the reactive power demands increase when the asynchronous machines are utilized in wind farms. So voltage levels drop down more, even beyond the lower limits of wind farms buses.

With the increase of wind power installed capacity in power systems, the variability of wind power generation causes variability of voltage level, particularly if integrated into the grid which might not be specifically designed to cater for the significant and possibly rapid load variations (compared with normal customer load variation) caused by highly variable wind power generation. Therefore, the regulatory measures are needed to maintain the voltage level in a specified range. However, the variability of wind power generation is not a low probability; this could result in an increased requirement for reactive power ancillary services to manage voltage control [5].

System Stability. In the power system with high wind power penetration, the transient stability, voltage stability and frequency stability are all influenced by the wind power integration not only because the injection of wind power will change the power flow distribution, transferred power of each transmission line and total inertia of the whole power system, but also because the wind turbine generators perform differently in either steady-state or transient-state compared with the conventional synchronous machine [6].

For current operation of wind farms, protections usually cut off the connections between wind farms and the grid when great disturbances occur. This is equivalent to arouse new generators tripping disturbance after the great disturbances. So the transient stability in such moment is very crucial, especially when large-scale wind farms are integrated.

Compared the variable-speed wind turbine based on the doubly-fed induction generator (DFIG) with the fixed-speed wind turbine based on the induction motor, the former is more robust after short-circuit failures and can strengthen system stability with keeping enough stability margin. However, wind power integration may also make the system transient stability worse due to the grid structure. Therefore, transient stabilities of different power systems should be analyzed respectively. The fixed-speed wind turbine absorbs the reactive power when outputting the active power. The whole demand of a wind farm for the reactive power is considerable, which lead to the decrease of the voltage stability in the area near PCC. On the contrary, the variable-speed wind turbine based on DFIG has certain ability to control the reactive power. According to different operation and control schemes, this wind turbine can absorb or output the reactive power to control the voltage, which benefits the voltage stability. The voltage stability is also related with the short-circuit capacity of PCC, transmission line ratios of $\mathrm{R} / \mathrm{X}$ and reactive compensation methods utilized of wind farms.

When the accident of frequency dropping sharply occurs, the inertia of the power system is decisive of the change rate of frequency dropping. The lower the total inertia is, the faster the frequency drops. In the power system, any decrease of the inertia response is dangerous for severe frequency accidents. Different types of wind turbines have different frequency response features. The rotor speed of the fixed-speed wind turbine couples strongly with the system frequency so that when the frequency declines the wind turbine slows down to release part of its kinetic energy and provides the inertia response. On the contrary, the variable-speed wind turbine based on DFIG cannot provide the inertia response for the grid when the frequency is changing due to its decoupling of the active and reactive power control, which cannot help the grid to slow down the change rate of the frequency. After conventional plants are replaced by wind farms consisting of variable-speed wind turbines, the decrease of the inertia is very disadvantageous for the frequency stability under the condition of small system capacity and low load for the system with high wind power penetration.

Power Quality. Fluctuations in the wind power and the associated power transport (AC or DC), have direct consequences to the power supply quality. As a result, large voltage fluctuations may result in voltage variations outside the regulation limits, as well as violations on flicker and other power quality standards.

During the continuous operation and switching operation, wind turbine causes voltage fluctuation and flicker, which are main concerns of unfavorable influence of wind power generation on power 
quality of the grid. For wind turbine of variable-speed and constant-frequency, harmonic issue caused by converters should also be considered.

The grid interferences of wind turbines or wind farms have different causes, which are mostly turbine-specific. Average power production, turbulence intensity and wind shear refer to causes that are determined by meteorological and geographical conditions. All the other causes are attributed not only by the characteristics of the electrical components, such as generators, transformers and so on, but also by the aerodynamic and mechanical behavior of the rotor and drive train. The turbine type (i.e. variable versus fixed speed stall versus pitch-regulated) is of major importance to the power quality characteristics of wind turbines and wind farms.

Variable-speed wind turbines can control the power output of the inverter system by pitch control, thus smoothing power fluctuations as well as power peaks. Thus the power peaks lie within the range of the rated power. Instantaneous power peaks of fixed-speed wind turbines often exceed rated power by $30 \%$ or more, even in the case of pitch-controlled fixed-speed wind turbines. The number of wind turbines on a wind farm is important for smoothing power peaks.

Recently, variable-speed turbines are equipped with self-commutated inverter systems, which are mainly PWM inverters, using an insulated gate bipolar transistor. This type of inverter has the advantage that both the active power and reactive power can be regulated. However, it has the disadvantage that it produces harmonic currents. Therefore, filters are necessary to reduce the harmonics.

Short-circuit Capacity. The majority of the wind power farms tend to be constructed remote from the load center, which means that electrical distance between them and the other part of the power system is rather long. There is a common sense that long electrical distance makes voltage variation bigger but short circuit problem smaller [7].

However, wind power farms will be able to give more and more significant effects on the calculation of short circuit current in future power system operation.

The reason is twofold. One is the above stated fact that wind power generation site is usually apart from the conventional electrical power center. It implies that the distribution of short circuit current might make a drastic change, leading to a completely different short circuit capacity map. The other reason is the fact that more and more wind power generation today are particularly in the form of so called large-scale wind farms (hundreds of megawatts). In wind farms a substantial number of individual units are connected together, and the total generation capacity will greatly rise up.

Wind farms have great influence on the short circuit capacities of adjacent nodes while the nodes far apart from PCC are little influenced [8]. Therefore, when wind farms with huge capacities are integrated into the grid, capacities of adjacent transformers and switches may need to be increased. It should be studied further how to determine the influence of wind power generation on the short circuit current ratings of existing electrical equipments on the network.

System Reserve. No power source is $100 \%$ reliable. Even huge nuclear stations can he tripped out of service by protection or withdrawn to allow maintenance to he carried out. However, the consequences due to a tripping can be managed using spinning reserve and, during maintenance, alternative generation can be in service. While large generators remain on a utility grid, the spinning reserve cover which these demand will continue to cover variations in wind power generation. However, if wind energy is to become the main source of energy, the loss of it, which is dependent on the weather and the variability of the wind resource, cannot be dealt with in this way. If strong interconnections between wind farm sites exist, the geographic spread of generators would be expected to lead to a smoothing out of the energy delivered to the network once a certain number have been developed.

Meanwhile, system load forecast will potentially become less accurate as the amount of wind power generation increases, which in turn influence power system operational scheme and unit commitment. In the case of generation reserve forecasts, this could translate into higher reserve level requirements to cover uncertainties in the availability of wind power generation [9].

The short-term output variations of large-scale wind farms are not so considerable, about $3 \%$ of 
the installed capacities, due to their interactions. By the process of the wind power generation growing rapidly, power systems need not only positive reserves when actual wind powers are lower than the predicted values, but also negative reserves when wind powers are higher than the predicted values.

Frequency Regulation. In the power system, the frequency is an indicator of the balance or imbalance between production and consumption. For normal power system operation, the frequency should be close to its nominal value. Fluctuations of wind farms outputs could lead to additional imbalances. As wind turbines use other generation technologies than conventional power plants, they have a limited capability of participating in primary frequency control in the same way conventional generators do. Since wind cannot be controlled, power production at normal frequency would be intentionally kept lower than possible in order for the wind farm to be able to provide secondary control at under frequencies. Danish regulations additionally require wind farms to take part in frequency control (secondary control) in island conditions. In order to control power system frequency within defined standards, grid corporations require some power plants to provide frequency control ancillary services. However, as the total amount of wind power generation increases, variation in its output will have a more significant impact on the frequency [10].

The penetration of intermittent wind generation will increase the need for continuous frequency regulation. Wind generation could also increase the need for frequency response, although this depends upon the extent to which wind generation will be able to satisfy future Grid Code requirements. The effect of rapid variations in the output of individual wind generators will be relatively minor, as the level of correlation between the fluctuations of the individual wind farms will be small in the time horizon considered (several seconds to a minute). It follows that the direct impact of wind generation on dynamic frequency control service may be small. However, as the volume of wind generation increases, the error in the forecast of its aggregate output will also increase.

This will increase dispatch errors, which will be neutralized by automatic governor actions, placing additional requirements on continuous frequency response.

Protection. As for any proposed expansion of a utility grid, there are problems to be addressed with each application for connection. For connection to the distribution system, the proposed generation must have a relatively low capacity. In general, that part of the network is radial and the protection would be graded to expect fault current to flow outwards from the hulk supply point. Introduction of a generator into the radial network means that fault current can now be supplied which does not flow in the expected direction.

A detailed check of the protection settings with the proposed generator in the network model is therefore required. The results of this check may show that the protection will function quite adequately as it is. At the other extreme, it may show that the existing relays cannot protect the network while the new generation and a redesign of the protection are necessary.

The power flows between wind farms and the grid are bidirectional, which should be considered during the protections design and configuration.

Whatever kind of generators are adopted in wind turbines, integration of a wind farm will increase the fault level of grid and furthermore affect the relay settings of original protection devices of grid. It is probably necessary to add new protection devices and/or modify the relay settings of original protection devices. Particularly if wind farms are connected into distribution networks, overloading of circuit breakers may occur with the increase of installed capacity of wind farm.

\section{Conclusion}

Wind energy has come a long way since the prototypes of just a few years ago, and it will probably continue to advance over the next twenty years. There are a number of issues associated with integration of wind power in system operation and development.

Although penetration of wind power generation may displace significant amount of energy produced by conventional plants, concerns are focused on the interaction between wind power 
generation and the grid. This paper has provided an overview of the influence of wind power generation on power systems and recommended corresponding countermeasures to handle these issues in order to accommodate wind power generation in power systems.

\section{Acknowledgement}

This study is supported by the project of public welfare of Zhejiang province (Grant No. 2015C31072).

\section{References}

[1] Burton T,Sharpe D, Jenkins N,et al. Wind energy handbook[M]. Chichester: John Wiley \& Sons Ltd, 2001.

[2] Bowden G J, Barker P R, Shestopal V O, et al. Weibull distribution function[J]. Wind Engineering, 1983, 7(2):85-98.

[3] Zhang Kun, Li Chun-sheng, Mao Cheng-xiong. Power Control of Directly-driven Wind Generation Systems With Battery/Ultra-capacitor Hybrid Energy Storage[J], Proceedings of the CSEE, 2012, 32(25):99-108.

[4] J Liang Hui, Shi Wei-wei. Study of Instantaneous Current Control for Multiple Boost Converter in Wind Energy Conversion System[J], Transactions of China Electrotechnical Society, 2011, 26(4):86-92.

[5] Chen Tie-jun, Ning Mei-feng, Wang Zhao-cai. Hybrid control of variable-speed variable-pitch system in wind power generation[J], Electric power automation equipment, 2013, 33(2):14-19.

[6] Chi Yongning, Liu Yanhua, Wang Weisheng, et al. Study on impact of wind power integration on power system [J]. Power System Technology, 2007, 31(3):77-81.

[7] Ackermann T. Wind power in power systems[M], Chichester:John Wiley \& Sons Ltd, 2005.

[8]Chen Ze. Wind Power in Electrical Distribution Systems[J], Transactions of China Electrotechnical Society, 2013,28( 5):1-14.

[9] Strbac G, Shakoor A, Black M, et al. Impact of wind generation on the operation and development of the UK electricity systems[J], Electric Power Systems Research, 2007, 77(9): 1214-1227.

[10] Eriksson K, Liljegren C, Sobrink K. HVDC light experiences applicable for power transmission from offshore wind power parks[EB/OL]. http://www.abb.com. 